\title{
Dietary uptake pattern affects bioaccumulation and biomagnification of hydrophobic organic compounds in fish
}

\footnotetext{
Xinghui Xia ${ }^{1}$, HaOtian Wang ${ }^{1}$, Derek C.G. MuiR ${ }^{2}$

${ }^{1}$ School of Environment, Beijing Normal University, Beijing 100875,China,xiaxh@bnu.edu.cn

${ }^{2}$ Aquatic Contaminants Research Division, Environment and Climate Change Canada, Burlington, ON, L7S 1A1 Canada

Biomagnification of hydrophobic organic compounds (HOCs) increases their eco-environmental risks. Lipid-water partitioning theory cannot fully address biomagnification. Here, we gained mechanistic insights on biomagnification of HOCs in fish from the kinetics. A new kinetic model describing the accumulation of HOCs in fish via waterborne and dietary uptake was established to consider discrete dietary uptake, while the conventional model regards dietary uptake as a continuous process. Bioaccumulation of the deuterated polycyclic aromatic hydrocarbons (PAHs-d10) in zebrafish was conducted with a passive dosing method in order to maintain the freely dissolved concentrations at $\mathrm{ng} \mathrm{L}^{-1}$. The PAH-d10 steady-state concentrations in zebrafish did not increase significantly with additional dietary uptake from Daphnia magna (with $\mathrm{ng} \mathrm{g}^{-1}$ PAHsd10), but they increased markedly with additional dietary uptake from spiked fish food (with $\mu \mathrm{g} \mathrm{g}^{-1}$ PAHs-d10). The results indicated that the intake amount of the PAHs-d10 played an important role in affecting their steady-state concentrations. Moreover, the peak concentrations in zebrafish after each dietary uptake make conventional kinetic model regarding dietary uptake as a continuous process underestimate the risks of the PAHs-d10. Considering the randomness of predation in natural situations, the Monte Carlo simulation showed that the probability distributions of biomagnification of the PAHs-d10 in zebrafish via trophic transfer from Daphnia magna increased with the dietary uptake frequency and amount of the PAHs-d10. The discontinuity and randomness of dietary uptake results in unpredictability of biomagnification, which may explain the among-aquatic ecosystem differences and provide new insight for analyzing experimental and field bioaccumulation data in the future.
} 